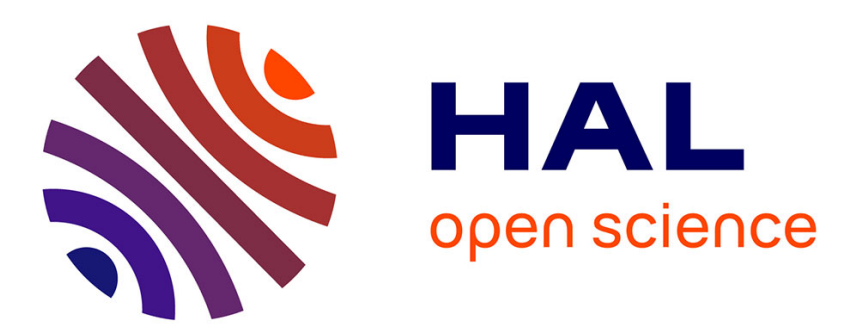

\title{
Surface Effects in the Pressure-Induced Structural Transformation of a ZnO Nanorod
}

\author{
Johannes Frenzel, Gotthard Seifert, Dirk Zahn
}

\section{To cite this version:}

Johannes Frenzel, Gotthard Seifert, Dirk Zahn. Surface Effects in the Pressure-Induced Structural Transformation of a ZnO Nanorod. Journal of Inorganic and General Chemistry / Zeitschrift für anorganische und allgemeine Chemie, 2009, 635 (12), pp.1773. 10.1002/zaac.200900260 . hal-00515810

\section{HAL Id: hal-00515810 \\ https://hal.science/hal-00515810}

Submitted on 8 Sep 2010

HAL is a multi-disciplinary open access archive for the deposit and dissemination of scientific research documents, whether they are published or not. The documents may come from teaching and research institutions in France or abroad, or from public or private research centers.
L'archive ouverte pluridisciplinaire HAL, est destinée au dépôt et à la diffusion de documents scientifiques de niveau recherche, publiés ou non, émanant des établissements d'enseignement et de recherche français ou étrangers, des laboratoires publics ou privés. 


\section{Surface Effects in the Pressure-Induced Structural Transformation of a ZnO Nanorod}

\begin{tabular}{|r|l|}
\hline Journal: & Zeitschrift für Anorganische und Allgemeine Chemie \\
\hline Manuscript ID: & zaac. 200900260.R1 \\
\hline Wiley - Manuscript type: & Article \\
\hline Date Submitted by the \\
Author: & $29-$ Jul-2009 \\
\hline Complete List of Authors: & $\begin{array}{l}\text { Frenzel, Johannes; TU Dresden, Physikalische Chemie } \\
\text { Seifert, Gotthard; TU Dresden, Physikalische Chemie } \\
\text { Zahn, Dirk; MPI CPfS }\end{array}$ \\
\hline Keywords: & structural transformations, nucleation processes, surface effects \\
\hline
\end{tabular}

\section{S) ScholaroNE" \\ Manuscript Central}




\title{
Surface Effects in the Pressure-Induced Structural Transformation of a ZnO Nanorod
}

\author{
Johannes Frenzel ${ }^{1,2}$, Gotthard Seifert ${ }^{2}$ and Dirk Zahn ${ }^{1}$ * \\ ${ }^{1}$ Dresden, Max-Planck-Institut für Chemische Physik fester Stoffe \\ ${ }^{2}$ Dresden, Technische Universität, Institut für Physikalische Chemie \\ * zahn@cpfs.mpg.de
}

Bei der Redaktion eingegangen am

Dedicated to Prof. Matrin Jansen on the occasion of his $60^{\text {th }}$ birthday. 


\begin{abstract}
We report on molecular dynamics simulations of an atomistic zinc oxide nanorod model embedded in a pressure medium. The transformation of the nanorod from the wurtzite to the rocksalt structure is investigated using both 'brute-force' over-pressurization and transition path sampling at a much lower pressure. The latter approach yields the underlying mechanisms at more realistic conditions and reveals the interplay of the morphogenesis of the rocksalt-type structure domains with shape deformation of the overall nanorod. Surface effects are observed to not only influence dedicated nucleation sites but also impose control mechanisms to phase growth and to specific shape deformation.
\end{abstract}




\title{
Introduction
}

While for many decades the investigation of high-pressure polymorphs has focused on bulk materials, the advance of nanocrystal research has recently opened a new perspective to this field. After the first demonstration of the size dependence of first order solid-solid transitions [1], Alivisatos and coworkers elaborated a series of further phenomena related to the finite size of nanoparticles. The latter include critical delimiters for nanocrystal metastability [2], and nanorod fracture [3]. Moreover, the importance of shape deformation for exploring the mechanisms of wurtzite-rocksalt transformations was demonstrated [4].

Complementing the experimental efforts, molecular dynamics simulations represent a promising tool, in particular, for the investigation of the transformation mechanisms. While this approach offers femto-second resolution at the atomistic level of detail, an important limitation to the exploration of first-order transitions originates from the short simulation time scales accessible. To enforce the crossing of nucleation barriers within pico to nano-seconds, conventional approaches refer to a considerable degree of overpressurization. By now, this method was applied in a large series of simulation studies, including the wurtzite $\rightarrow$ rocksalt transition of nano crystal models $[5,6]$.

\begin{abstract}
A typical shortcoming of such 'brute-force' molecular dynamics simulations is related to the strong driving of the process kinetics which -for studies in the bulk- were shown to severely bias the mechanistic analyses [7]. To overcome this limitation, we recently
\end{abstract}


demonstrated the suitability of the transition path sampling (TPS) approach for tackling nucleation barrier crossing in solid-solid transformations [8].

Moreover, in 2007, Dellago and coworkers pioneered TPS simulations of solid-solid transitions in nanoparticles at the example of a $\mathrm{Cd}_{528} \mathrm{Se}_{528}$ nanocrystal [9]. In concordance to previous TPS simulations related to bulk CdSe [10], also for the nanocrystal strong evidence for a layer sliding mechanism was obtained. Dellago and coworkers could furthermore observe preferential nucleation at the surface, followed by domain growth via subsequent (100) layer sliding [9]. Though limited by the small size of the nanoparticle model, some insights into the competition of the up and down shifting of (100) planes could be obtained.

In the present work, the interplay of shape deformation with structural motif nucleation and domain growth is explored in further detail. From this we wish to explore the role of interface/surface energy minimization and its implications to the nanoparticle shape after transforming to the rocksalt structure. This requires the modeling of sufficiently large nanocrystal models to reduce the tendency towards coupling of the atomic displacements inherent to particularly small nanoparticles. Moreover, the role of different (over)pressurization is explored by contrasting the TPS approach to 'brute-force' molecular dynamics simulations. 


\section{Simulation Details}

Our studies are based on a periodic simulation system comprising of a nanorod embedded in a solution of spherical particles acting as a medium to exert hydrostatic pressure (fig. 1). The particles of the surrounding medium intact via Lennard-Jones (lj) type potentials, only. To hinder crystallization of the pressurization medium, the solution was prepared of two types of particles $\mathrm{P}_{1 \mathrm{j}}{ }^{1}$ and $\mathrm{P}_{\mathrm{lj}}{ }^{2}$ with different van-der-Waals radii of 5 and $6 \AA$, respectively. This choice was motivated by performing 1 nanosecond molecular dynamics simulations of the pure $\mathrm{P}_{\mathrm{lj}}{ }^{1} / \mathrm{P}_{\mathrm{lj}}{ }^{2}$ liquid which did not exhibit crystallization at a pressure of $10 \mathrm{GPa}$.

The $\mathrm{ZnO}$ force-field was chosen from Lewis and Catlow [11]. We performed structure optimizations at 0 Kelvin of the wurtzite and the rocksalt modification and calculated the coexistence pressure as $5.8 \mathrm{GPa}$. A full list of all interaction potentials is provided in table $1[11,12]$. The molecular dynamics simulations were performed at constant pressure (9 to $6 \mathrm{GPa})$ and constant temperature $(300 \mathrm{~K})$ using a time-step of 0.1 fs.

Within the TPS scheme $[13,14]$, the molecular dynamics simulations are focused on relatively short time-sketches in which the process of interest occurs, and largely ignore the 'waiting' time required for the observation of spontaneous nucleation events. Starting from an initial transformation pathway needed as a prerequisite, TPS creates further transition routes in an iterative manner. This corresponds to a Monte-Carlo sampling of the trajectory space of pathways. The first transformation pathway was obtained by 
applying sufficiently large pressure and exploring the atomic movement from a 14 ps molecular dynamics simulations. Several pressure values were tested and at $9 \mathrm{GPa}$ the transformation was found to occur spontaneously. However, as known from the Oswald rule of phases, the extreme driving forces used in such 'brute-force' approaches typically lead to the fastest, rather than the thermodynamically preferred mechanistic route. Nevertheless, by using such pathways as a starting point to TPS iterations, realistic transition routes may be obtained after convergence of the Monte-Carlo sampling in trajectory space $[15,16,17]$. The TPS procedure works at a much larger range of driving forces (for processes such as reactions in solution no external driving is needed at all). Performing TPS at $9 \mathrm{GPa}$ only provides variations of the trajectory illustrated in figure 1 and does not allow additional insights. However, by lowering the simulation pressure to 6 GPa we could investigate the phase transition at conditions for which 100 ps straightforward molecular dynamics simulations did not show spontaneous transformation. More importantly, the reduction to more realistic external driving leads to mechanistic changes which may be too subtle to be observed from over-pressurization. The mechanistic analysis as described in the following is based on 800 TPS iterations of which 109 were identified as successful transformation routes. During the automated sampling procedure, the average (nearest-neighbor, $r_{i j} \leq 2.5 \AA$ ) coordination number was used as an order parameter for discriminating different crystal structures. 


\section{Results}

In anology to previous findings for bulk $\mathrm{ZnO} / \mathrm{CdSe}$ and $\mathrm{CdSe}$ nanorods $[9,10]$, the wurtzite $\rightarrow$ rocksalt transformation was found to occur via a two-step process comprising of a wurtzite $\rightarrow \mathrm{h}-\mathrm{MgO}$ transition and subsequent transformation to the rocksalt structure. The former step reflects a simple axial compression along the [001] direction. In the following, we focus on the second step, i.e. the $\mathrm{h}-\mathrm{MgO} \rightarrow$ rocksalt transition for which a peculiar interplay of phase nucleation and growth with the shape and structure of the nanorod was observed. This phenomenon originates to the sliding of (100) layers occurring during the transformation which -depending on their implementation- may give rise to drastic shape changes.

For the simulation runs performed at $9 \mathrm{GPa}$, almost all layer sliding events may be characterized by alternating parallel and anti-parallel (100) layer shifts. As a consequence, the hexagonal shape of the (001) faces of the nanorod is largely preserved and a rocksalt structure type nanorod of a hexagonal prismatic habitus is formed (fig.1). The corresponding surface structure displays a large density of imperfections such as steps and notches. Both are unfavorable in potential energy and should give rise to different nanocrystal morphologies. We explored profiles of the potential energy for the rocksalt structure type nanocrystals obtained from transition path sampling at $6 \mathrm{GPa}$. Figure 2 clearly shows the importance of the surface structure for the local potential energy profile. Moreover, the disfavoring of steps and notches may be identified as a surface effect acting on the $\mathrm{h}-\mathrm{MgO} \rightarrow$ rocksalt transformation. 
While this subtle effect is overrun at $9 \mathrm{GPa}$ (i.e. the conditions at which the starting pathway \#1 was prepared), at more modest driving of the transition process ( $6 \mathrm{GPa})$, the surface effect takes a significant role leading to a different type of transformation routes. This evolution of transition pathways gives rise to drastic changes in the nanocrystal shape aiming at more smooth surfaces. Along this line, the overall mechanism of (100) layer sliding is preserved, whilst the sequence of up and down shifts is changed considerable from the antiparallel shuffling observed for bulk models [10,18]. Indeed, only the very first onset of rocksalt domain nucleation occurs via antiparallel layer sliding moves (fig. 3). Once a rocksalt domain -including patches of the nanorod surfaces- is established, the systems tendency to avoid surface stress controls the direction of the layer shifting during further phase growth. The latter involves a series of parallel displacements which give rise to the formation of smooth nanorod surface patches.

Accordingly, the final shape of the nanorod much depends on the morphogenesis of phase nucleation and growth. As the h-MgO type nanorod corresponds to an ideal hexagonal prism with smooth surfaces, the very initial stage of nucleation, i.e. the shifting of three adjacent (100) layers, requires the formation of ridges irrespective of parallel or antiparallel layer shifting. The surface effect discussed above hence does not yet apply and the atomic displacements follow the same mechanism as observed for bulk models with periodic boundaries. Indeed, from the perspective of a very initial phase nucleus the embedding nanocrystal of a few nm dimensions appears quite akin to a bulk structure. This picture changes drastically as soon as a first patch of rocksalt-type 
nanocrystal surface is established. After the shifting of the first three (100) layers, all further displacements of adjacent layers follow the direction of the previous one. As a consequence, the initial nucleation site is 'marked' by a kink in the nanorod surface, whilst further phase growth involves smooth surface patches, only. 


\section{Conclusion}

In conclusion, we reported on transition path sampling molecular dynamics simulations of the wurtzite to rocksalt transformation of zinc oxide nanorod model embedded by a pressure medium. By choosing sufficiently large nanorod dimensions and by discrimination of different pressure conditions, a detailed mechanistic picture of phase nucleation and growth is elaborated. The latter is governed by a simple and widely transferable rule, i.e. the minimization of stress by formation of favorable (smooth) surfaces. The study confirms the dominant role of surfaces (and associated surface free energy) on determing the transformation pathwayand we expect our findings to be of general relevance to displacive structural transitions in nanocrystals.

\section{Acknowledgements}

We thank Jamshed Anwar for helpful discussions and acknowledge high-performance computing resources provided by the ZIH Dresden. 


\section{References}

[1] S.H. Tolbert, A.P. Alivisatos, Science 1994, 265, 373.

[2] C-C Chen, A.B. Herhold, C.S. Johnson, A.P. Alivisatos, Science 1997, 276, 398.

[3] D. Zaziski, S. Prilliman, E.C. Scher, M. Casula, J. Wickham, S.M. Clark, A.P. Alivisatos, Nano Letters 2004, 4, 943.

[4] J.N. Wickham, A.B. Herold, A.P. Alivisatos, Phys. Rev. Lett. 2000, 84, 923.

[5] B.J. Morgan, P.A. Madden, Nano Letters 2004, 4, 1581.

[6] B.J. Morgan, P.A. Madden, Phys.Chem.Chem.Phys. 2006, 8, 3304.

[7] D. Zahn, J. Solid State Chem. 2004, 177, 3590.

[8] D. Zahn, S. Leoni, Phys. Rev. Lett. 2004, 92, 250201.

[9] M. Grünwald, C. Dellago, P.L. Geissler, J. Chem. Phys. 2007, 127, 154718.

[10]D. Zahn, Y. Grin, S. Leoni, Phys. Rev. B 2005, 72, 064110.

[11] G.V. Lewis, C.R.A. Catlow, J.Phys. C: Solid State Phys. 1985, 18, 1149.

[12] S.L. Mayo, B.D. Olafson, W.A. Goddard III, J. Phys. Chem. 1990, 94, 8897.

[13]P.G. Bolhuis, C. Dellago, D. Chandler, Faraday Discuss. 1998, 110, 421.

[14] C. Dellago, P.G. Bolhuis, F.S. Csajka, D. Chandler, J. Chem. Phys. 1998, 108, 1964.

[15]D. Zahn, Phys. Rev. Lett. 2004, 92, 40801.

[16]D. Zahn, Phys. Rev. Lett. 2004, 93, 227801.

[17]D. Zahn, J. Chem. Theory Comput. 2006, 2, 107.

[18] S.E. Boulfelfel, S. Leoni, Phys. Rev. B 2008, 78, 125204. 


\begin{tabular}{c|c|c|c|c}
$\mathbf{i}-\mathbf{j}$ & $\mathbf{A} / \mathbf{~ k J m o l}^{-1}$ & $\mathbf{\rho} / \AA$ & $\mathbf{C} / \AA^{\mathbf{6}} \mathbf{~ k J m o l}^{-1}$ & $\mathbf{q}_{\mathbf{i}} \cdot \mathbf{q}_{\mathbf{j}} / \mathbf{e}^{\mathbf{2}}$ \\
\hline $\mathbf{Z n} * \mathbf{Z n}$ & 0.0 & 0.0 & 0.0 & 4 \\
\hline $\mathbf{O} * \mathbf{O}$ & 2196420.11 & 0.149 & 10825.65 & 4 \\
\hline $\mathbf{Z n} * \mathbf{O}$ & 67568.65 & 0.3372 & 0.0 & -4
\end{tabular}

\begin{tabular}{c|c|c}
$\mathbf{i}$ & $\boldsymbol{\sigma}_{\mathrm{ii}} / \AA$ & $\varepsilon_{\mathrm{ii}} / \mathbf{~} \mathbf{J} \mathbf{~ m o l}^{-\mathbf{1}}$ \\
\hline $\mathbf{P}_{\mathbf{l j}}{ }^{\mathbf{1}}$ & 5 & 1.0 \\
\hline $\mathbf{P}_{\mathbf{l j}}{ }^{2}$ & 6 & 1.0 \\
\hline $\mathbf{Z n}^{2+}$ & 0.69 & 0.05857742 \\
\hline $\mathbf{O}^{2-}$ & 3.2 & 1.04602529
\end{tabular}

Tables 1: For the $\mathrm{Zn}^{2+. .} \mathrm{O}^{2-}$ interactions the Buckingham-type potential of Lewis and Catlow (top) was applied. To model all other interactions Lennard-Jones type potentials and standard mixing rules were used. 


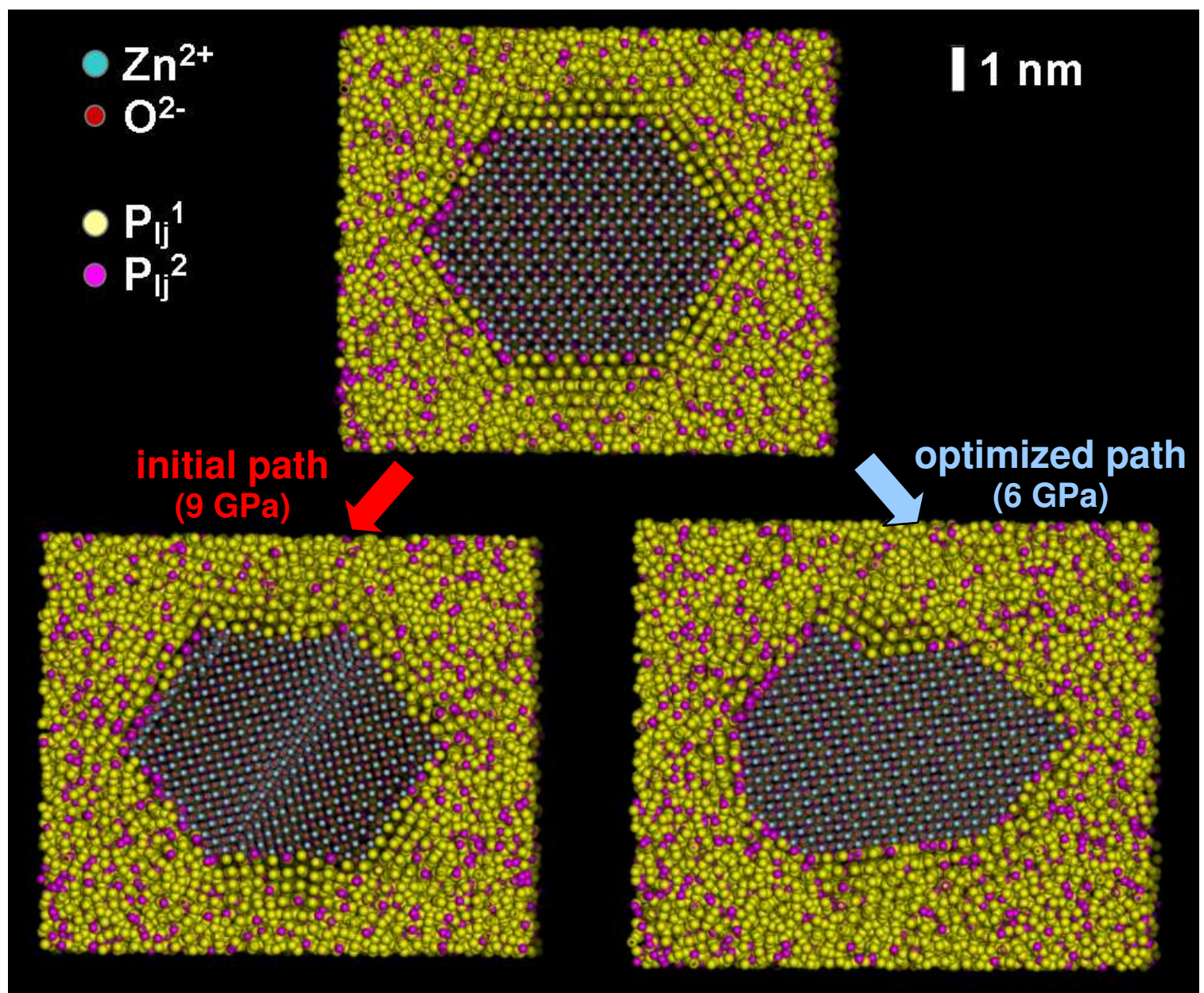

Fig. 1

Illustration of the simulation model before and after the wurtzite $\rightarrow$ rocksalt transition of the $\mathrm{ZnO}$ nanorod. The left hand side corresponds to the initial pathway as obtained from brute-force molecular dynamics simulations using high pressure. At the lower right, the final configuration of an optimized transformation route referring to more realistic pressure is shown. The initial $\mathrm{ZnO}$ nanorod is modeled as an ideal hexagonal prism of $25200 \mathrm{Zn}^{2+}$ and $\mathrm{O}^{2-}$ ions. The pressure medium is mimicked by a 1:4 liquid mixture of 25000 soft spheres. 


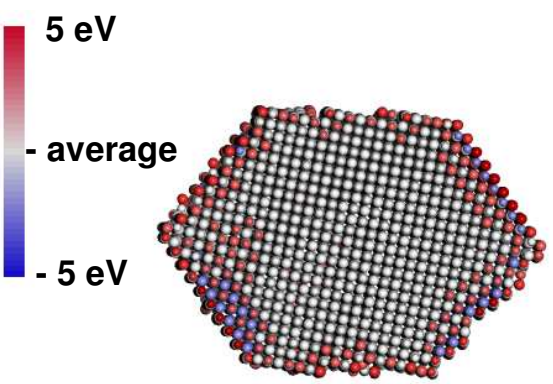

\# 1

$<E_{\text {atom }}>=-27.04 \mathrm{eV}$

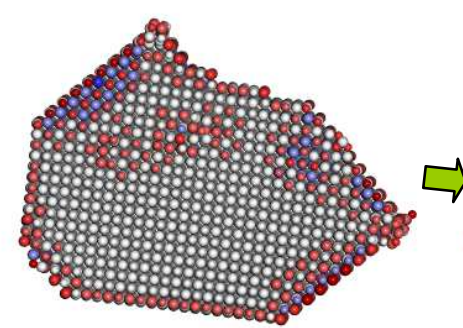

\#25

$<E_{\text {atom }}>=-27.063 \mathrm{eV}$

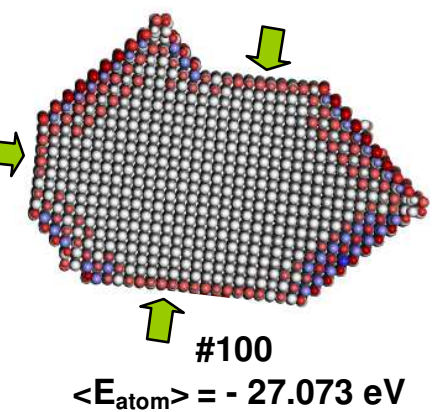

Fig. 2

Energy distribution of rocksalt type structures as obtained from transition path sampling at $6 \mathrm{GPa}$. For all atoms the incorporation energy $\Delta \mathrm{E}_{\mathrm{i}}=\mathrm{E}_{\text {nanorod }}-\mathrm{E}_{\text {atom i removed }}$ was compared to its average value of $-27 \mathrm{eV}$. To illustrate local stress, the atoms are colored according to $\Delta \Delta \mathrm{E}_{\mathrm{i}}=\Delta \mathrm{E}_{\mathrm{i}}+27 \mathrm{eV}$. In the course of the optimization of wurtzite $\rightarrow$ rocksalt transition routes, the total energy of the nanorod model decreases and the overall shape experiences considerable changes. Along this line, notched surfaces are reorganized in favor of smooth (100) (indicated by green arrows) and (110) faces. This gives rise to specific implementations of the layer shifting mechanism as shown in figure 3. All energy values refer to the isolated nanorod. The overall shape effect comprises the surface energy (of the nanorod in vacuum) and an interface energy term which depends on the choice of the pressure medium. 


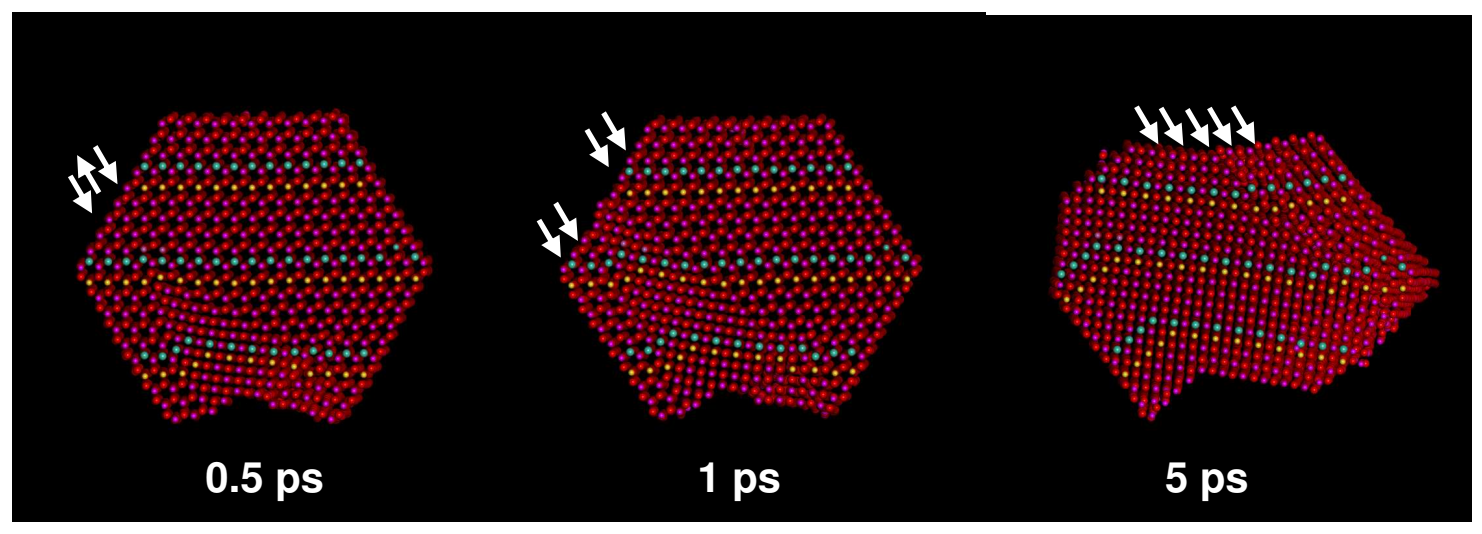

Fig. 3

Snapshots of an optimized wurtzite $\rightarrow$ rocksalt transition route illustrating the nucleation and growth of the rocksalt structure and its interplay with the shape of the nanorod model. Along the horizontal direction, several atomic rows are highlighted to help the visualization of the (100) layer shifting mechanism and its interplay with shape deformation. To avoid the formation of notched surfaces, antiparallel layer shifting as observed for the beginning of the nucleation process is disfavored. Later stages of phase growth are found to follow a uniform sliding of layers. 


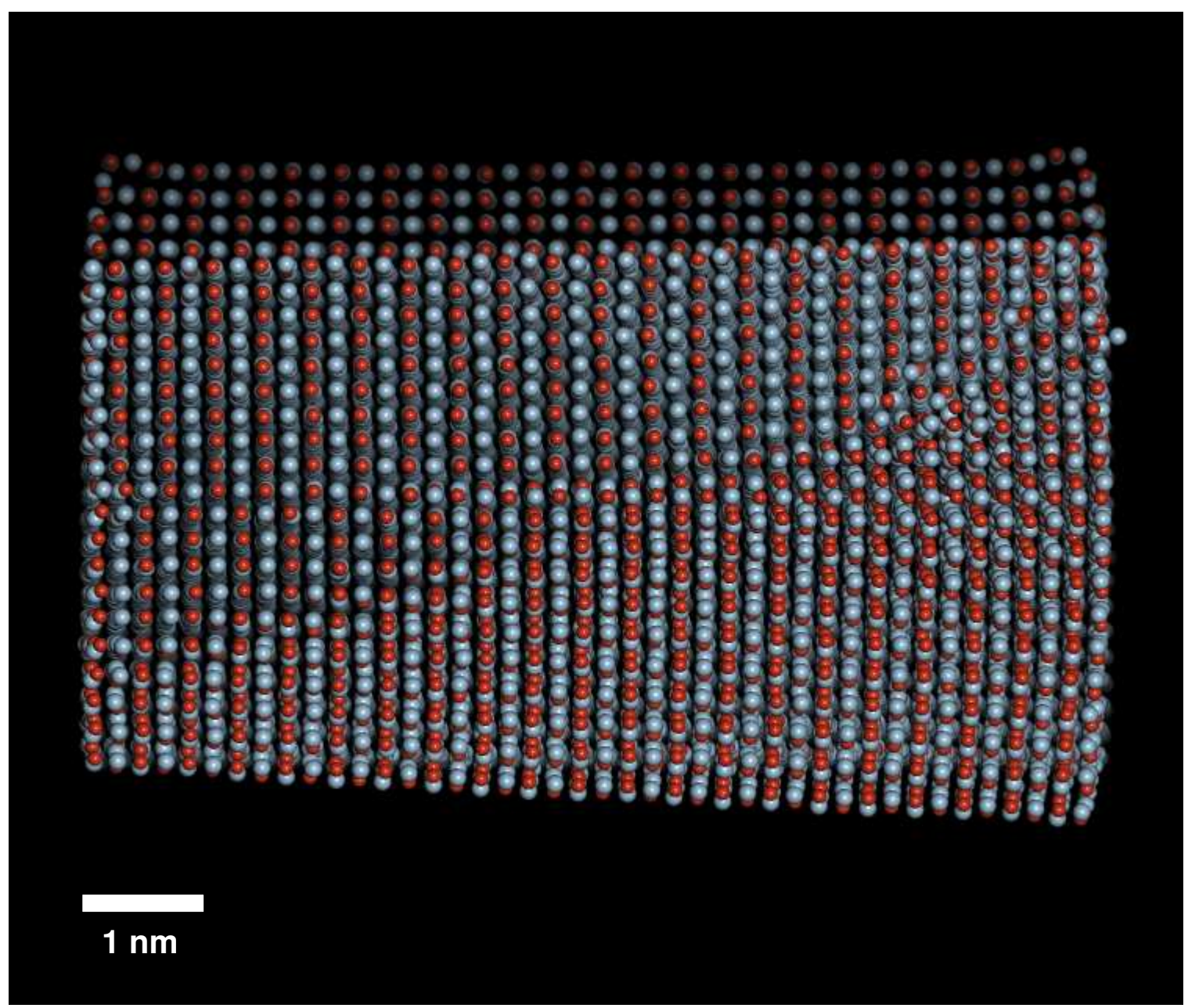

Fig. 4:

Same snapshot as shown in the center of figure 3 (1 ps), but illustrating the wurtziterocksalt transition along the c-axis of the nanorod. The atomic shifting occurs at fastest rate along the c-axis, and leads to the shuffling of layer patches rather than individual columns. 


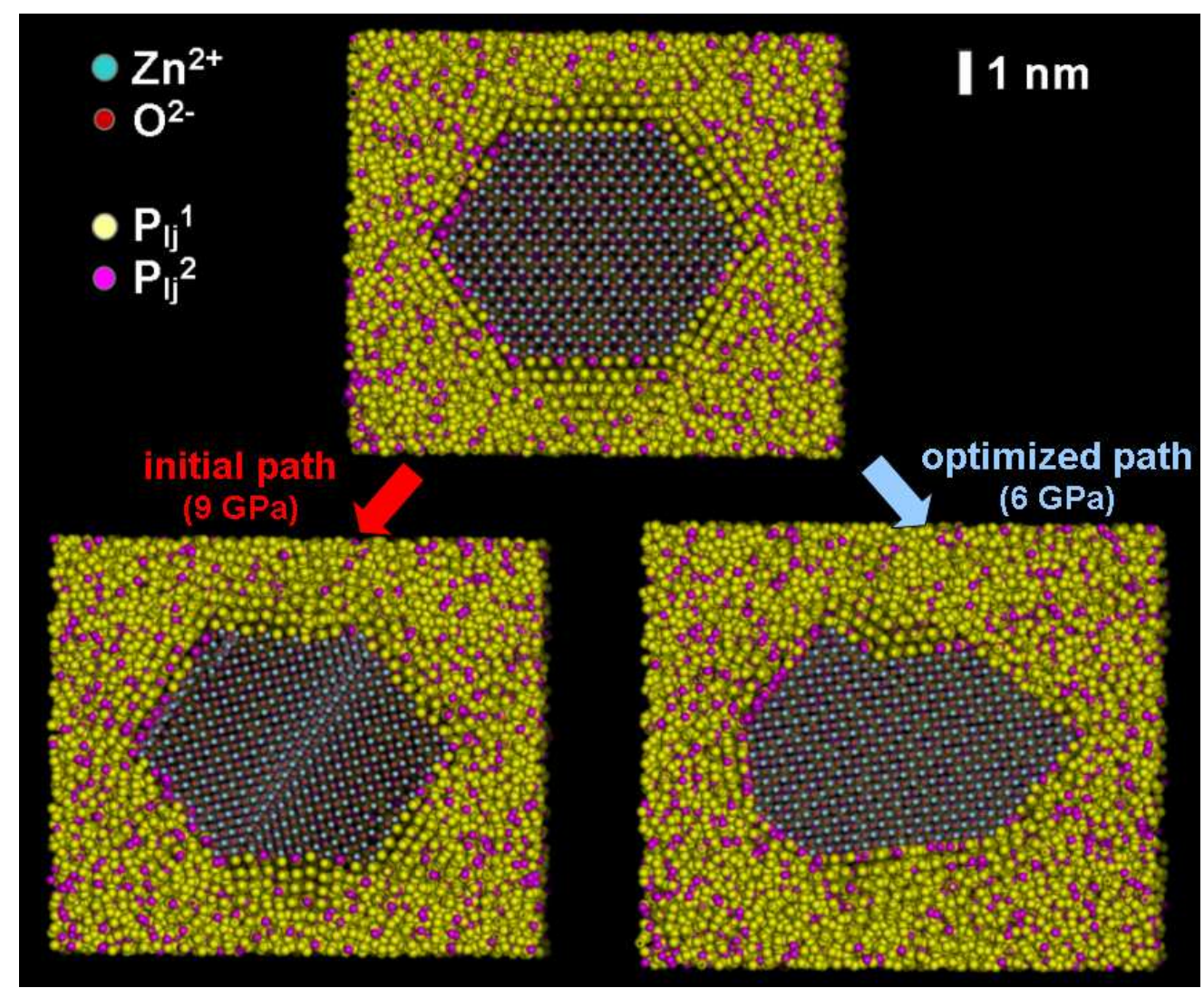

$302 \times 249 m m(72 \times 72$ DPI) 


1
2
3
4
5
6
7
8
9
10
11
12
13
14
15
16
17
18
19
20
21
22
23
24
25
26
27
28
29
30
31
32
33
34
35
36
37
38
39
40
41
42
43
44
45
46
47
48
49
50
51
52
53
54
55
56
57
59
60
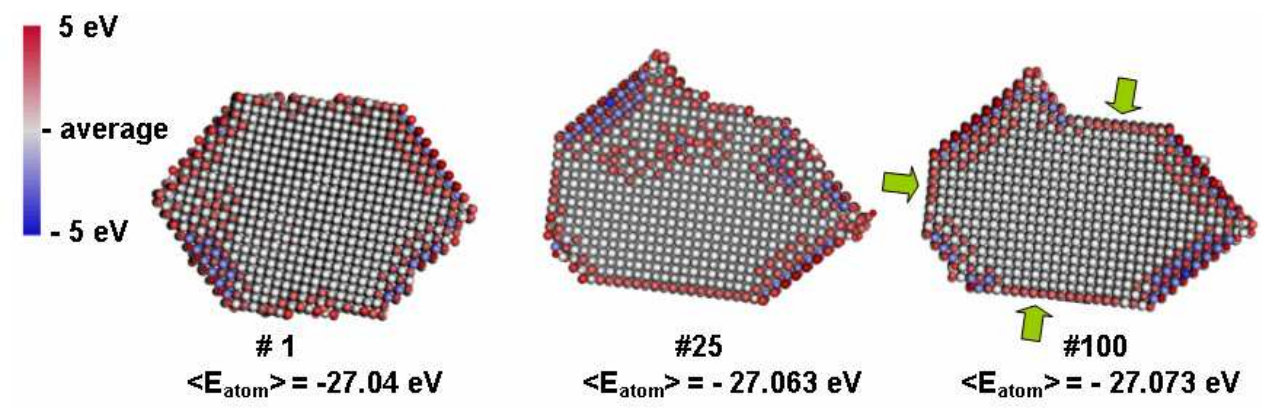

$315 \times 113 \mathrm{~mm}(72 \times 72$ DPI $)$ 


1
2
3
4
5
6
7
8
9
10
11
12
13
14
15
16
17
18
19
20
21
22
23
24
25
26
27
28
29
30
31
32
33
34
35
36
37
38
39
40
41
42
43
44
59
45
46
47
48
49
50
51
52
54
56

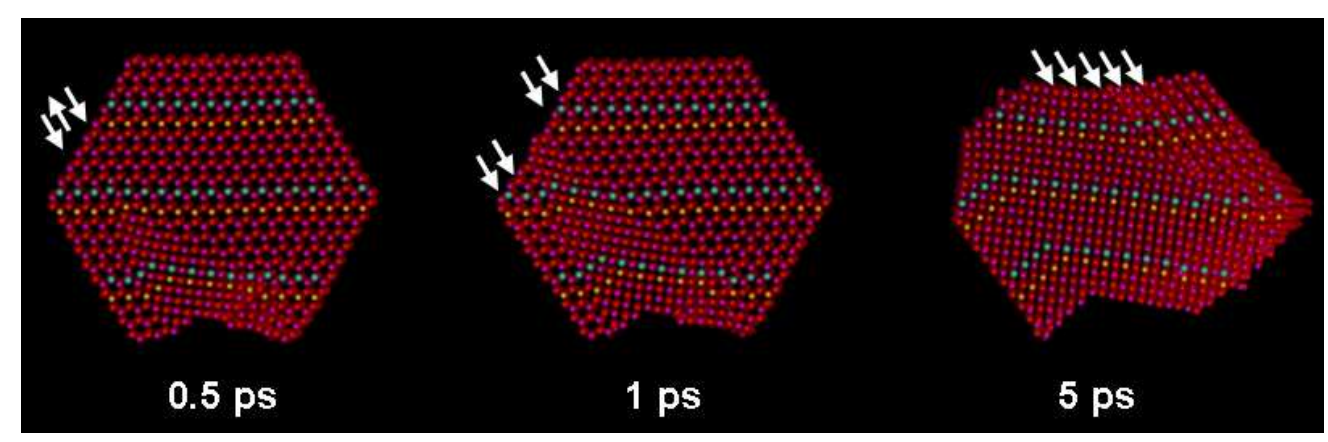

$285 \times 90 \mathrm{~mm}(72 \times 72 \mathrm{DPI})$ 


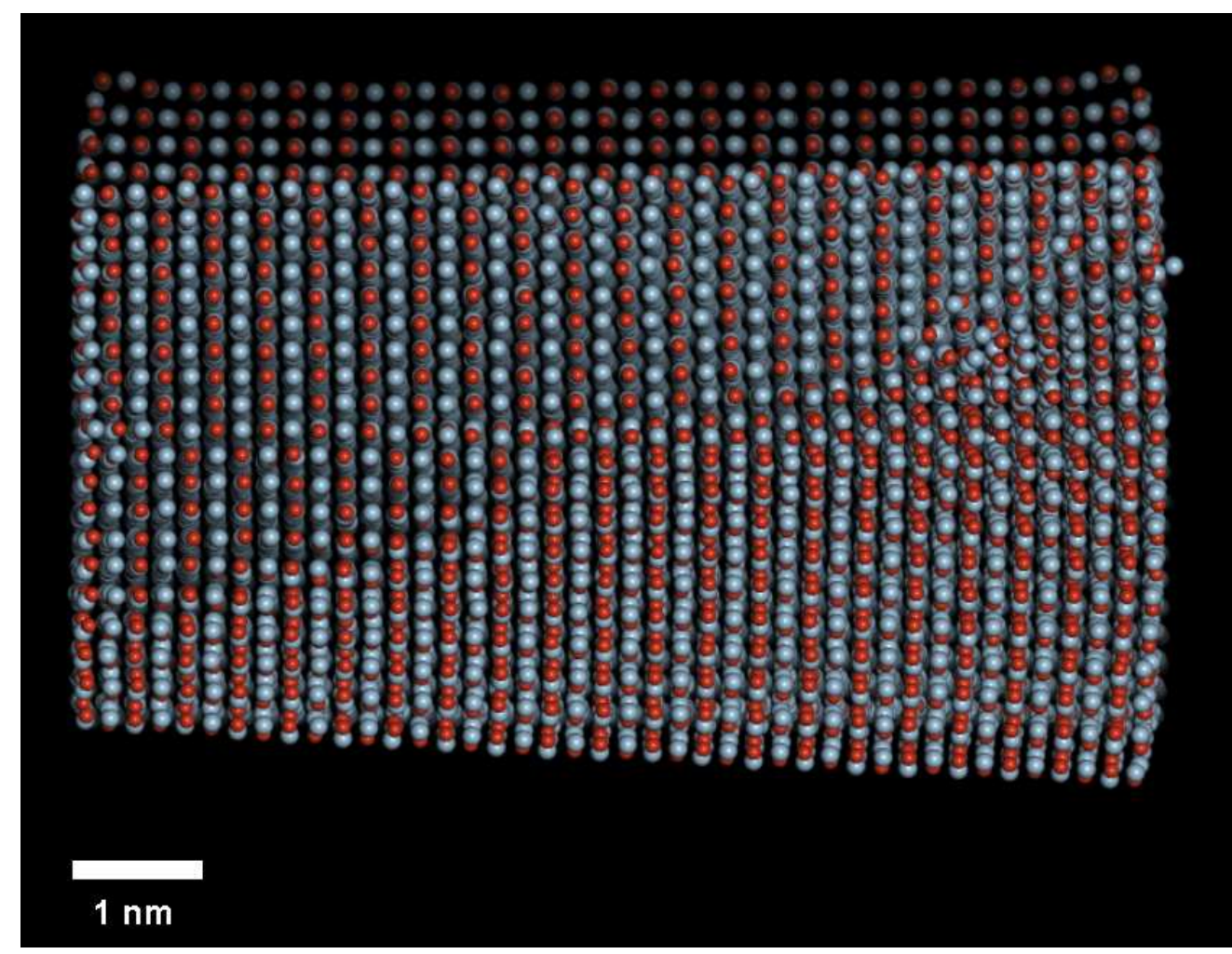

$295 \times 227 \mathrm{~mm}(72 \times 72 \mathrm{DPI})$

Wiley-VCH 